\title{
EVALUACIONES ECONÓMICAS DE TECNOLOGÍAS SANITARIAS: UNA PERSPECTIVA GLOBAL PARA SU APLICACIÓN EN AMÉRICA LATINA
}

\author{
Manuel Espinoza1,a, Marianela Castillo-Riquelme ${ }^{2, b}, V_{i c t o r} Z^{1} a a^{1, c}$
}

\begin{abstract}
RESUMEN
Fenómenos como el aumento progresivo del gasto en salud y el envejecimiento poblacional han obligado a los distintos países a considerar metodologías económicas que permitan obtener un mayor beneficio sanitario dentro de un contexto de recursos limitados. El presente artículo describe los componentes básicos a considerar en una evaluación de tecnología sanitaria, analiza el proceso de toma de decisión en un análisis de costo efectividad y reporta como dicha metodología ha sido implementada en América Latina y en el resto de mundo.
\end{abstract}

Palabras clave: Evaluación Económica; Evaluación de las tecnologías sanitarias; América Latina (fuente: DeCS BIREME).

\section{ECONOMIC EVALUATIONS OF HEALTH TECHNOLOGIES: A GLOBAL PERSPECTIVE FOR THEIR IMPLEMENTATION IN LATIN AMERICA}

\begin{abstract}
Phenomena as the progressive increase of health expenditure and the population aging have lead many countries to consider economic methodologies in order to obtain bigger sanitary benefits in contexts of limited resources. This article describes the basic components to consider in a health technology assessment, it analyses the process of decision making with cost-effectiveness analysis and reports how this methodology has been widely implemented in Latin America and the rest of the world.
\end{abstract}

Key words: Health economics; Health technology assessment; Latin America (source: MeSH NLM).

\section{INTRODUCCIÓN}

El aumento de los costos en salud es una realidad compartida por un gran número de países, fenómeno que con frecuencia se ha asociado con la adopción de nuevas tecnologías. Esta realidad ha llevado a distintos organismos públicos y privados a buscar mecanismos que permitan generar una mayor eficiencia en el sistema de salud. Es en este sentido que la evaluación económica $(E E)$ ha tomado un fuerte impulso durante las últimas décadas y, cada vez más, se integra a los requerimientos básicos de calidad, seguridad y eficacia solicitados a toda intervención sanitaria ${ }^{(1)}$.

En términos prácticos, una EE se define como un análisis comparativo de cursos alternativos de acción, en términos de sus costos y consecuencias ${ }^{(2)}$. Palmer et al. clasificaron las evaluaciones económicas de tecnologías sanitarias en tres tipos que se diferencian, principalmente, en la forma que estiman los beneficios: costo-beneficio, costo-efectividad y costo-utilidad ${ }^{(3)}$. Los estudios de costo-beneficio involucran la medición tanto de los costos, como de los beneficios, en términos monetarios. El estimador final representa una ganancia monetaria neta, que puede ser comparada con la producida por otras intervenciones de salud. A pesar de su amplia aplicación en distintos sectores de la economía, los estudios de costo-beneficio son, generalmente, poco aceptados en salud, dado que la necesidad de poner un valor económico a ganancias en salud genera con facilidad conflictos morales o religiosos. Los estudios de costoefectividad, por otra parte, miden los beneficios sanitarios en unidades naturales, muchas veces clínicas, como la disminución de la presión arterial en milímetros de mercurio, en el caso de terapia antihipertensiva, o la disminución de peso en kilos en el tratamiento de la obesidad. En este caso, el estimador final expresa los costos y los beneficios en distintas unidades, lo que solo permite determinar razones de costo-efectividad de distintas intervenciones para una determinada enfermedad

Centre for Health Economics, University of York. York, Reino Unido.

2 Departamento de Economía de Salud, Ministerio de Salud de Chile. Santiago, Chile.

a Médico especialista en Economía de la Salud; ${ }^{b}$ Economista de la Salud; c Médico especialista en Salud Pública

Recibido: 09-07-11 Aprobado: 14-09-11 
o escenario de salud. Finalmente, los estudios de costoutilidad proveen una solución al problema anteriormente descrito, ya que su unidad de beneficio incluye aspectos genéricos relacionados con la cantidad y calidad de la salud. Esto permite la comparación transversal de distintas intervenciones para distintas enfermedades en cuanto a sus índices de costo-utilidad. Algunas de las unidades de beneficio más reportadas en la literatura, en los estudios de costo-utilidad, son los años de vida ajustados por calidad (AVAC o QALY por su sigla en inglés) y los años de vida ajustados por discapacidad (AVAD o DALY por su sigla en inglés) ${ }^{(4)}$. Si bien existen claras diferencias metodológicas entre los tres tipos de evaluaciones económicas descritos, existe una tendencia a referirse genéricamente a todas ellas como evaluaciones de costo-efectividad.

\section{ANÁLISIS DE COSTO EFECTIVIDAD}

El proceso de decisión respecto de la incorporación de nuevas tecnologías al arsenal preventivo, diagnóstico o terapéutico de un sistema de salud, enfrenta una serie de preguntas que deben ser sistemáticamente abordadas. La primera es si la nueva tecnología cumple con el perfil de seguridad y eficacia, consideraciones mínimas para plantear su uso. Segundo, en qué medida la eficacia reportada podría variar cuando la tecnología se implementa en un escenario real, en otras palabras, cuál es la efectividad de la nueva intervención. Tercero, dada la escasez de recursos disponibles para atender las necesidades de salud de la población, el decisor debe preguntarse si vale la pena pagar por la nueva tecnología.

La primera de estas preguntas es habitualmente abordada basados en la información disponible de estudios clínicos experimentales, principalmente ensayos clínicos controlados. Adicionalmente, estos son sintetizados utilizando métodos de revisiones sistemáticas y metanálisis. Sin embargo, este tipo de información cuenta con importantes limitaciones como es, por ejemplo, el tiempo de seguimiento de dichos estudios, que no siempre logra ser el tiempo horizonte que interesa para efectos del problema de decisión, algo particularmente relevante en enfermedades crónicas. Aun cuando los ensayos clínicos controlados presentan altos índices de validez interna, las decisiones poblacionales requieren mayor validez externa, es decir, que los resultados puedan ser aplicados a toda la población objetivo. A esto se suma el hecho de que muchas veces la evidencia existente está limitada por la disponibilidad de estudios que hayan incluido comparaciones directas entre las intervenciones por evaluar. Por ejemplo, en el análisis de la incorporación de estatinas al arsenal terapéutico, interesa evaluar el efecto relativo de todas las estatinas disponibles comparadas entre ellas, sin embargo, la evidencia obtenida de ensayos clínicos provee solo algunas comparaciones directas ${ }^{(5)}$.

El análisis de costo efectividad se ha desarrollado conforme a la necesidad de ofrecer apoyo a las decisiones de salud. En este contexto, elementos como el uso de modelos de decisiones y métodos de síntesis de evidencia de base bayesiana son un considerable avance en cuanto entregan una representación más realista del problema, considerando toda la evidencia relevante disponible ${ }^{(6)}$. Adicionalmente, los métodos como comparaciones indirectas y mixtas, proveen la oportunidad de sintetizar todas las fuentes de evidencia existentes simultáneamente. Esto permite estimar indirectamente -primero- el efecto de tratamiento de aquellos comparadores para los que no existe evidencia empírica y, -segundo- su incertidumbre asociada (7). Por otro lado, los modelos analíticos como árboles de decisión y modelos de Markov no solo son útiles para una mejor estimación de la efectividad de las intervenciones y, por tanto, mayor validez externa, sino también para extrapolar los resultados ofrecidos por la evidencia actual al tiempo horizonte que el decisor requiera ${ }^{(8)}$.

El objetivo central del análisis de costo-efectividad es establecer un comparador relativo de costos y efectos que le permita al decisor enfrentar la pregunta si el gasto en esta nueva tecnología representa un correcto uso de los recursos disponibles por el sistema de salud ${ }^{(9)}$. Sin embargo, no es el presupuesto la única restricción que debe ser considerada en el proceso de decisión. La información o evidencia disponible también constituye una restricción para la toma de decisiones ${ }^{(10)}$. Así, el decisor enfrenta dos preguntas que, aunque simultáneas, son distintas, la primera es si dada la información disponible vale la pena adoptar la nueva tecnología y, la segunda, es si la obtención de mayor información a través de nueva investigación es costo efectiva para el sistema de salud ${ }^{(11,12)}$.

Para responder ambas preguntas, es que el análisis de costo-efectividad ha visto importantes avances en los últimos veinte años respecto de los métodos de representación de la incertidumbre ${ }^{(13-15)}$. Esta caracterización de la incertidumbre es relevante en cuanto permite valorar la probabilidad de tomar una decisión incorrecta y sus consecuencias, ambos componentes fundamentales para tomar una decisión con información limitada. Dicha aproximación contrasta con la clásica adoptada en epidemiología clínica, la cual se ancla en los clásicos criterios de la inferencia estadística. Lejos de utilizar un valor $p$ en el contexto de una dócima de hipótesis (procedimiento que permite contrastar dos hipótesis bajo ciertas consideraciones y tomar una decisión respecto de ellas), o un intervalo de confianza, o de credibilidad 
(su contraparte bayesiana), el marco de costo efectividad rechaza los criterios arbitrarios de la inferencia estadística adoptando una aproximación más racional que combina probabilidades y consecuencias ${ }^{(16)}$.

Finalmente, estos métodos no solo ofrecen una aproximación racional al problema actual de decisión sino que también permiten enfrentar la pregunta si resulta apropiado conducir mayor investigación que permita llenar los vacíos de incertidumbre ${ }^{(17,18)}$. Es así como los métodos de valor de la información ${ }^{(19,20)}$ y valor del muestreo ${ }^{(21)}$ informan tanto del tipo de investigación que debe ser realizada como de la muestra máxima que debiera ser utilizada basado en un criterio de eficiencia de asignación de recursos, más que en clásicas reglas de la inferencia estadística.

En consecuencia, los métodos asociados al análisis de costo-efectividad han avanzado considerablemente en los últimos años, sin embargo, aún quedan muchas áreas donde se requiere mayor investigación metodológica; por ejemplo, los métodos para incorporar sistemáticamente heterogeneidad y análisis de subgrupos está en sus inicios ${ }^{(22)}$, lo mismo ocurre con las consideraciones de equidad en este tipo de evaluaciones ${ }^{(23)}$. Por otro lado, los métodos de valor de la información y de muestreo han tenido un lento ingreso, en buena parte debido a la complejidad de su implementación computacional pero también por la falta de comprensión por parte de los decisores. Sin embargo, a pesar de lo anterior, es probable que en los próximos años este tipo de estudios sean cada vez más requeridos dada la necesidad de los sistemas de salud en optimizar la asignación de sus limitados recursos.

\section{EVALUACIÓN ECONÓMICA EN SALUD EN EL CONTEXTO INTERNACIONAL}

Un creciente número de países, especialmente desarrollados, han incorporado la evaluación económica formalmente en sus procesos de asignación de recursos en salud. En muchos casos la EE forma parte de un proceso de evaluación de tecnologías sanitarias (ETE$\mathrm{SA}$ ) que busca informar decisiones de reembolso (especialmente medicamentos), cobertura de intervenciones o incorporación de tecnologías sanitarias. En el Reino Unido la creación del National Institute for Health and Clinical Excellence (NICE) en 1999, dependiente del Servicio Nacional de Salud, fue probablemente la piedra angular en la formalización de las EE en la toma de decisiones en salud para Inglaterra y Gales. Recientemente se ha descrito el proceso que sigue el NICE tanto para la elaboración de guías de práctica clínica como más ampliamente para la adopción (o rechazo) de una determinada tecnología ${ }^{(24)}$.
Los países se han organizado de distintas formas para hacer frente a la demanda de las EE respondiendo básicamente a las características de sus sistemas de salud (tanto desde el financiamiento como geográficamente). Las ETESA son llevadas a cabo por una variedad de organizaciones tanto del sector público como privado, así como por comités asesores y cuerpos regulatorios ${ }^{(25)}$. Además de Inglaterra, Canadá, Suecia y Alemania ya cuentan con reconocidas agencias que producen evaluaciones de alta calidad para de tomadores de decisiones. Estas son la Canadian Agency for Drugs and Technologies in Health (CADTH), el Swedish Council for Health Technology Assessment (SBU), y la German Agency of Health Technology Assessment at the German Institute for Medical Documentation and Information (DAHTA@DIMDI) y el Institute for Quality and Efficiency in Health Care (IQWiG). Sin embargo, con mayor frecuencia las organizaciones encargadas de tomar las decisiones finales en salud encargan las evaluaciones tanto a las agencias de ETESA (públicas o privadas) como al sector académico.

En el caso de Latinoamérica, región donde los servicios de salud se organizan con frecuencia por la combinación de sector público, seguridad social y sector privado; tanto las EE como las ETESA son un tema nuevo con grados de desarrollo variables entre los países. La limitada evidencia sobre la realización y uso de las EE en la toma de decisiones en salud en Latinoamérica fue reportada en 2005 por Iglesias et al. ${ }^{(26)}$, quienes en una revisión sistemática analizaron las publicaciones de la región desde 1982 en adelante. Se identificaron 554 artículos de los cuales solo 93 eran EE. Los países que lideraban las publicaciones eran Brasil, México, Argentina y Colombia, aunque la mayoría de los estudios se habían realizado en colaboración con instituciones de países desarrollados. La mayoría de los artículos eran estudios de costo-efectividad, un significativo número de ellos presentaron problemas de calidad metodológica. Finalmente, los autores concluyen que las decisiones sobre implementación de programas de salud pública siguen siendo de tipo político, históricas o de acuerdo a referentes geográficos.

Un estudio similar, pero publicado recientemente, reporta algunos aspectos relacionados con la generalización y transferabilidad de resultados de las EE dentro de la región ${ }^{(27)}$. En una revisión sistemática, se identificaron 521 artículos (período 1980-2004), de los cuales 72 calificaron como EE. Nuevamente, Brasil, Argentina y México lideraban las publicaciones. Los autores encontraron que si bien algunos aspectos de la evaluación se desarrollaban óptimamente (formulación de preguntas, descripción de población, intervenciones y comparadores), en general, la calidad del reporte era 
pobre. Se evidenciaron análisis deficientes, así como falta de adherencia a las directrices en temas tales como la aplicación de tasas de descuento y la realización del análisis incremental. Los autores concluyen que no es posible evaluar las potencialidades para transferabilidad, ya que esta última se asocia directamente con la calidad de los estudios.

El desarrollo de la ETESA en Latinoamérica ha sido investigado por Arellano et al. quien identificó seis agencias de ETESA en la región ${ }^{28)}$. En base a un análisis de 236 documentos que publicaron estas instituciones entre enero de 2000 y marzo de 2007, el autor informa que la mayoría (82 \%) eran documentos cortos, $14 \%$ eran revisiones sistemáticas y solo $1 \%$ correspondían a ETESA completas. Entre otras características, los aspectos que evalúan estos documentos son la efectividad clínica (40\%), las prácticas actuales (29\%), los costos y efectividad (26\%) y otros resultados (6\%). El reporte indica que de entre los estudios que evalúan costos y efectividad solo $8 \%$ conduce EE completa. Los autores destacan el hecho de que en Latinoamérica falta un marco conceptual integral de ETESA.

Finalmente, el Organismo Andino de Salud (ORASCONHU) encargó un estudio en el marco de la Comisión Técnica subregional de Evaluación de Tecnologías Sanitarias. Los países incluidos en el estudio fueron Bolivia, Chile, Colombia, Ecuador y Perú ${ }^{(29)}$. El diagnóstico recogido por el estudio refleja que en el estado inicial del tema, existe una confusión entre gestión de tecnología y evaluación de esta. Se constató, además, que las normativas existentes en esta materia se refieren principalmente a medicamentos. La metodología consideraba el envío de encuestas a los profesionales puntos focales del tema en cada país. Al respecto, al ser consultados sobre los factores más relevantes en la ETESA, en primer lugar se menciona la calidad y seguridad y, en segundo lugar, los protocolos clínicos. La importancia de las EE, aparece en séptimo lugar (de un total de 12 factores). Los autores concluyen que desarrollar capacidades institucionales (principalmente de formación) es vital para el desarrollo de las ETESA en la región.

Uno paso importante en la institucionalización de la EE es la emisión de guías metodológicas a nivel nacional. En la región, Brasil, México, Colombia y Cuba ya han establecido los lineamientos esenciales para la EE a través de la emisión de guías metodológicas locales ${ }^{(30)}$. Se espera que varios países de la región avancen en este sentido. En Chile, el Ministerio de Salud (MINSAL) está asumiendo un rol activo en esta materia comprometiéndose con la emisión de la primera guía metodológica en EES.
En conclusión, la implementación de un proceso de evaluación de intervenciones sanitaria que considere EE tiene mucho por avanzar en América Latina. Formar capacidades de investigación así como sensibilizar a los decisores en esta materia, parecen ser la base del camino por recorrer. Adicionalmente, nuestra región presenta otros desafíos que impiden a los investigadores ir a la par con países desarrollados. Un ejemplo es la baja calidad de los datos y registros (especialmente referente a resultados de salud o efectividad), lo que constituye una limitación importante en varios países. Además, los estudios requieren adecuado financiamiento e investigadores capacitados, ambos recursos escasos en la región. Adicionalmente, hay que considerar las escasas posibilidades de formación académica en EE en la región, con ausencia de postgrados de nivel de magíster. Finamente, las publicaciones que revisten evidencia y podrían ser la base para la formación, siguen estando disponibles principalmente en idioma inglés en fuentes de difícil acceso.

\section{Contribuciones de autoría}

Todos los autores participaron en la concepción, diseño y redacción del manuscrito.

\section{Fuentes de financiamiento}

Este informe fue autofinanciado.

\section{Conflicto de interés}

Los autores declaran no tener conflictos de interés en la publicación de este artículo.

\section{REFERENCIAS BIBLIOGRÁFICAS}

1. Taylor RS, Drummond MF, Salkeld G, Sullivan SD. Inclusion of cost effectiveness in licensing requirements of new drugs: the fourth hurdle. BMJ. 2004;329(7472):972-5.

2. Drummond MF, O'Brien B, Stoddart GL, Torrance GW. Methods for the economic evaluation of health care programs. $2^{\circ}$ ed. Oxford: Oxford University Press; 1997.

3. Palmer S, Byford S, Raftery J. Economics notes: types of economic evaluation. BMJ. 1999;318(7194):1349

4. Arnesen T, Nord E. The value of DALY life: problems with ethics and validity of disability adjusted life years. BMJ. 1999;319(7222):1423-5.

5. Ara R, Pandor A, Stevens J, Rees A, Rafia R. Early highdose lipid-lowering therapy to avoid cardiac events: a systematic review and economic evaluation. Health Technol Assess. 2009;13(34):1-74.

6. Briggs A, Sculpher MJ, Claxton K, editors. Decision Modelling for Health Economic Evaluation. Gosport, Hampshire: Oxford University Press; 2006.

7. Ades AE, Sculpher M, Sutton A, Abrams K, Cooper N, Welton N, et al. Bayesian methods for evidence synthesis in cost-effectiveness analysis. Pharmacoeconomics. 2006;24(1):1-19. 
8. Briggs A, Sculpher M. An introduction to Markov modelling for economic evaluation. Pharmacoeconomics. 1998 Apr;13(4):397-409.

9. Drummond $\mathbf{M}, \mathbf{M c G u i r e} \mathbf{A}$, editors. Economic Evaluation in Health Care: merging theory with practice. 1 ra ed. New York: Oxford University Press; 2001.

10. Claxton K, Cohen JT, Neumann PJ. When is evidence sufficient? Health Aff (Millwood). 2005;24(1):93-101.

11. Claxton $K$, Sculpher M, Drummond M. A rational framework for decision making by the National Institute For Clinical Excellence (NICE). Lancet. 2002;360(9334):711-5.

12. Griffin S, Claxton K, Sculpher M. Decision analysis for resource allocation in health care. J Health Serv Res Policy. 2008;13 Suppl 3:23-30.

13. O'Hagan A, McCabe C, Akehurst R, Brennan A, Briggs $\mathbf{A}$, Claxton $\mathrm{K}$, et al. Incorporation of uncertainty in health economic modelling studies. Pharmacoeconomics. 2005;23(6):529-36.

14. Fenwick E, Claxton K, Sculpher M. Representing uncertainty: the role of cost-effectiveness acceptability curves. Health Econ. 2001;10(8):779-87.

15. Griffin SC, Claxton KP, Palmer SJ, Sculpher MJ. Dangerous omissions: the consequences of ignoring decision uncertainty. Health Econ. 2011;20(2):212-24.

16. Claxton K. The irrelevance of inference: a decision-making approach to the stochastic evaluation of health care technologies. J Health Econ. 1999;18(3):341-64.

17. Claxton K, Posnett J. An economic approach to clinical trial design and research priority-setting. Health Econ. 1996;5(6):513-24.

18. Ginnelly L, Claxton K, Sculpher MJ, Golder S. Using value of information analysis to inform publicly funded research priorities. Appl Health Econ Health Policy. 2005;4(1):37-46.

19. Claxton K. Bayesian approaches to the value of information: implications for the regulation of new pharmaceuticals. Health Econ. 1999;8(3):269-74.

20. Bojke L, Claxton K, Sculpher MJ, Palmer S. Identifying research priorities: the value of information associated with repeat screening for age-related macular degeneration. Med Decis Making. 2008;28(1):33-43.

21. Ades AE, Lu G, Claxton K. Expected value of sample information calculations in medical decision modeling. Med Decis Making. 2004;24(2):207-27.
22. Sculpher M. Subgroups and Heterogeneity in Cost-Effectiveness Analysis. Pharmacoeconomics. 2008;26(9):799.

23. Cookson R, Drummond $\mathbf{M}$, Weatherly $\mathbf{H}$. Explicit incorporation of equity considerations into economic evaluation of public health interventions. Health Econ Policy Law. 2009;4(Pt 2):231-45.

24. Castillo-Laborde C. Evaluaciones económicas en el proceso de toma de decisiones en salud: El caso de Inglaterra. Rev Med Chile. 2010;138(suppl 2):103-7.

25. Drummond MF, Schwartz JS, Jonsson B, Luce BR, Neumann PJ, Siebert U, et al. Key principles for the improved conduct of health technology assessments for resource allocation decisions. Int $\mathrm{J}$ Technol Assess Health Care. 2008;24(3):244-58; discussion 362-8.

26. Iglesias CP, Drummond MF, Rovira J. Health-care decision-making processes in Latin America: problems and prospects for the use of economic evaluation. Int J Technol Assess Health Care. 2005;21(1):1-14.

27. Augustovski F, Iglesias C, Manca A, Drummond M, Rubinstein A, Marti SG. Barriers to generalizability of health economic evaluations in Latin America and the Caribbean region. Pharmacoeconomics. 2009;27(11):919-29.

28. Arellano LE, Reza M, Blasco JA, Andradas E. A content analysis of Health Technology Assessment programs in Latin America. Int $\mathrm{J}$ Technol Assess Health Care. 2009;25(4):570-6.

29. Yglesias AB. Análisis comparativo de políticas y Normatividad Relacionada con la Evaluación de Tecnología Sanitaria en los países de la subregión andina. Lima: Organismo Andino de Salud / Convenio Hipólito Unanue; 2007.

30. Augustovski F, Garay OU, Pichon-Riviere A, Rubinstein A, Caporale JE. Economic evaluation guidelines in Latin America: a current snapshot. Expert Rev Pharmacoecon Outcomes Res. 2010;10(5):525-37.

Correspondencia: Víctor Zárate

Dirección: Centre for Health Economics, University of York, YORK, YO10 5DD, Reino Unido.

Teléfono: (+44)1904 321401

Correo electrónico: victor.zarate@york.ac.uk 